\title{
Transverse beam profile reconstruction using synchrotron radiation interferometry
}

\author{
L. Torino* and U. Iriso \\ ALBA - CELLS Synchrotron Radiation Facility, Carrer de la Llum 2-26, \\ 08290 Cerdanyola del Vallès (Barcelona), Spain \\ (Received 3 August 2016; published 27 December 2016)
}

\begin{abstract}
Transverse beam size measurements in new generation of synchrotron light sources is a challenging task due to their characteristic small beam emittances and low couplings. Since the late 1990s, synchrotron radiation interferometry (SRI) has been used in many accelerators to measure the beam size through the analysis of the spatial coherence of the synchrotron light. However, the standard SRI using a doubleaperture system provides the beam size projection in a given direction. For this reason, the beam shape is not fully characterized because information about possible transverse beam tilts is not determined. In this report, we describe a technique to fully reconstruct the transverse beam profile based on a rotating double-pinhole mask, together with experimental results obtained at ALBA under different beam couplings. We also discuss how this method allows us to infer ultrasmall beam sizes in case of limitations of the standard SRI.
\end{abstract}

DOI: 10.1103/PhysRevAccelBeams.19.122801

\section{INTRODUCTION}

Measurement and control of the particle beam emittance is a key ingredient to characterize the performance of any accelerator. Since the emittance itself is not a direct measurable parameter, most accelerators monitor its surrogate: the transverse beam size. The particle beam emittance is then inferred from the knowledge of the machine optical parameters [1].

Due to its nondestructive nature, synchrotron light sources have developed a variety of techniques to measure the transverse beam size based on the synchrotron radiation [2]. Among them, probably the most common emittance monitor is the x-ray pinhole camera due to its simplicity and reliability. This system was firstly used at European Synchrotron Radiation Facility (ESRF) [3], and at ALBA is the workhorse for transverse beam size measurements since its commissioning in 2011 [4].

Another widely used method to measure the transverse beam size is the synchrotron radiation interferometry (SRI), which is based on the analysis of the spatial coherence of the synchrotron light and has been used in accelerators since the late 1990s [5]. At ALBA, we have also upgraded our visible diagnostics beamline "Xanadu" to perform SRI. After fighting with several mechanical limitations like wavefront distortion due to mirror imperfections and

\footnotetext{
*1torino@cells.es

Published by the American Physical Society under the terms of the Creative Commons Attribution 3.0 License. Further distribution of this work must maintain attribution to the author $(s)$ and the published article's title, journal citation, and DOI.
}

air-vibrations [6], this beamline is currently producing reliable beam size measurements [7].

New generations of synchrotron light sources are characterized by their low emittance and low coupling beams, with horizontal emittances in the $\mathrm{nm}$ (or below) range. The beam coupling is usually controlled using skew quadrupoles and it typically ranges between $\sim[0.1,2] \%$. Changing the beam coupling not only provides a change in the vertical beam emittance, it also produces a change in the beam tilt. At ALBA, the SRI source point is located at Bending Magnet 1 (BM1). Figure 1 shows the (modeled) behavior of the beam tilt angle along the first ALBA quadrant for three different set of skew quadrupoles, providing three different beam couplings. Note that in dipole BM1, as well as in the other 3 dipoles in the sector, the beam tilt changes from about $-5^{\circ}$ to $-35^{\circ}$.

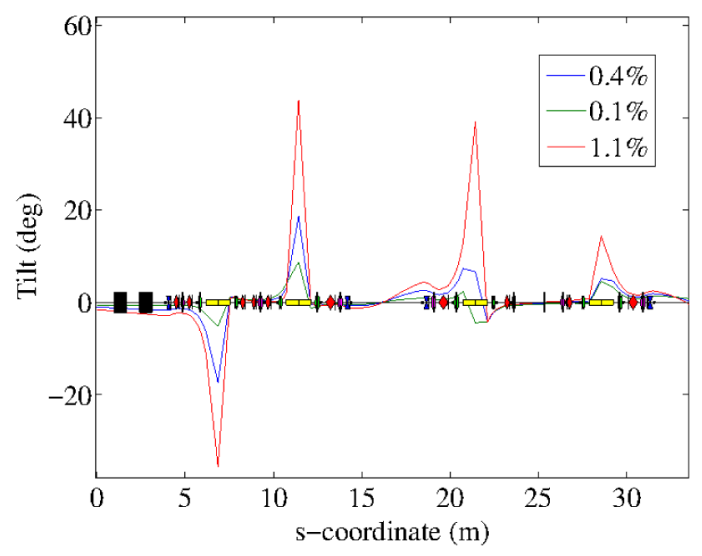

FIG. 1. Beam tilt angle for three different settings of the skew quads at ALBA in Sector 1. 
TABLE I. Nominal machine parameters at BM1, the source point of the diagnostic beamline Xanadu.

\begin{tabular}{lccc}
\hline \hline & $x$ & & $y$ \\
\hline Magnetic field $B$ & & $1.42 \mathrm{~T}$ & \\
$\beta(\mathrm{m})$ & 0.249 & & 24.741 \\
Emittance $\varepsilon(\mathrm{nm} \mathrm{rad})$ & 4.6 & & 0.023 \\
Coupling $K(\%)$ & & 0.5 & \\
Dispersion $D(\mathrm{~m})$ & 0.04 & & 0 \\
Energy spread $\frac{\Delta E}{E}$ & & 0.00105 & \\
Divergence $\sigma^{\prime}(\mu \mathrm{rad})$ & 136 & & 0.37 \\
Beam size $\sigma(\mu \mathrm{m})$ & 54 & & 23 \\
Tilt angle $(\mathrm{deg})$ & & -15 & \\
\hline \hline
\end{tabular}

Other parameters describing the electron beam at BM1 during normal machine operation are listed in Table I.

As opposed to the direct imaging techniques like the $\mathrm{x}$-ray pinhole camera, the standard SRI technique using a double-aperture system only provides the projected beam size along the axes of the apertures, and therefore information about the beam tilt change for different couplings is not available.

Beam tilt angle measurements were performed at Spring-8 using a 4-aperture interferometry [8]. At ALBA, this technique could not be successfully carried out because of experimental limitations, mainly related to air-vibrations. The work presented in this paper is a useful alternative: we show a method to reconstruct the full transverse beam profile using a rotating double-aperture system, which allows us to properly measure the beam size and relative beam-tilt angle.

It is worth mentioning that in parallel, recent (and independent) work in Spear-3 also used a rotating mask system to map the dependence of photon beam coherence on angle [9]. This paper goes further and describes a method to perform full transverse beam profile reconstruction, and how this "rotating mask" method can overcome limitations in measuring ultra-small beam sizes.

This paper is organized as follows: Section II shows the ALBA experimental setup, Sec. III recalls the main principles of the standard SRI. Section IV describes the mathematical basis which allows the beam profile reconstruction, and next the results under different couplings are provided (Sec. V). Finally, in Sec. VI, we show how this "rotating mask" method can overcome limitations in measuring ultra-small beam sizes.

\section{ALBA EXPERIMENTAL SETUP}

Synchrotron radiation is produced by a bending magnet, selected by a photon shutter and extracted through a motorized in-vacuum mirror. The mirror can be moved from 25 down to $7 \mathrm{~mm}$ from the orbit plane,

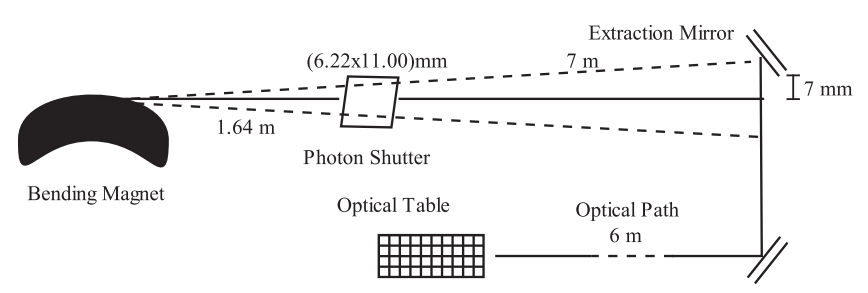

FIG. 2. Sketch ALBA diagnostic beamline, Xanadu. The synchrotron radiation beam is selected by a photon shutter located at $1.64 \mathrm{~m}$ from the source point. The in-vacuum extraction mirror is located $7 \mathrm{~m}$ downstream, $7 \mathrm{~mm}$ above the orbit plane. Seven other mirrors (already out of vacuum) bring the light from the tunnel to the Xanadu optical table.

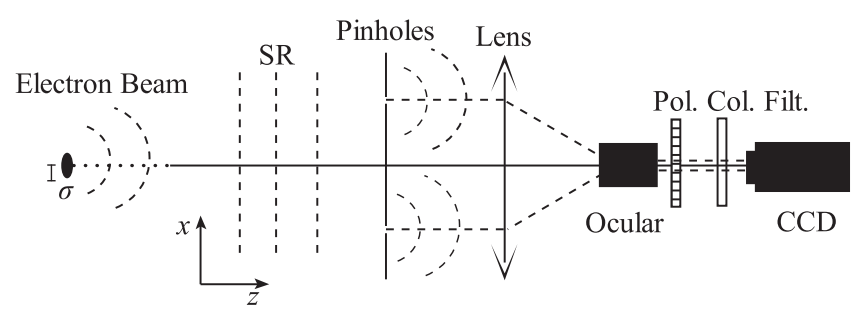

FIG. 3. Sketch of the SRI experimental setup: the synchrotron radiation (SR) produced by the beam passes through the double pinhole system and is imaged through a lens and an objective ocular to the CCD. The radiation polarization and the wavelength are selected through a polarizer (Pol.) and a color filter (Col. Filt.).

in order to not interfere with the x-rays. In this way only the upper lobe of the radiation is extracted and transported through 7 flat in air-mirrors to the beamline optical table. Here it is imaged by a double pinhole Young-like interferometry. A sketch of ALBA diagnostic beamline Xanadu [10], used for visible light diagnostic, is presented in Fig. 2.

The ALBA SRI system [7] consists of two pinholes, a lens to focus the interference fringes, a telescopic ocular to magnify the interferogram, a narrow band color filter and a polarizer to select wavelength and polarization of the light. The interferogram is finally captured by a Charge-Coupled Device (CCD) camera. A sketch of the SRI beam size measurement is presented in Fig. 3 and Table II lists the main characteristics of the optical elements used.

TABLE II. Characteristics of the ALBA SRI setup.

\begin{tabular}{lc}
\hline \hline Pinholes radius $a$ & $2.5 \mathrm{~mm}$ \\
Distance between pinholes $D$ & $16 \mathrm{~mm}$ \\
Radiation wavelength $\lambda$ & $538 \pm 10 \mathrm{~nm}$ \\
Lens focal distance $f_{l}$ & $500 \mathrm{~mm}$ \\
Ocular focal distance $f_{o}$ & $18 \mathrm{~mm}$ \\
Distance from the source point $L$ & $15 \mathrm{~m}$ \\
\hline \hline
\end{tabular}




\section{PRINCIPLES OF SRI}

The theory of the beam size measurements using SRI is explained in detail in Refs. [5,11]. Next, we give a brief overview of its main principles, and its limitations for a tilted beam.

The SRI is a wavefront-division type technique based on the analysis of the light spatial coherence. Using a doubleaperture mask, the system produces the interference of two polarized and quasimonochromatic light beamlets. At the detector plane, the interference is produced along the axis $(x)$ parallel to the double-aperture system, and when the apertures are pinholes of radius $a$, spaced by a distance $D$, the intensity of the interference pattern is described by [5]:

$I(x)=I_{0}\left\{\frac{\mathrm{J}_{1}\left(\frac{2 \pi a x}{\lambda f}\right)}{\left(\frac{2 \pi a x}{\lambda f}\right)}\right\}^{2} \times\left\{1+V \cos \left(\frac{2 \pi D x}{\lambda f}+\varphi\right)\right\}$,

where $I_{0}$ is the light intensity, $\lambda$ is the radiation wavelength, $f$ is the focal length of the imaging system (lens plus ocular, see Table II), and $\varphi$ is the phase of the interferogram fringes, which depends on the position of the pinholes with respect to the electron beam orbit plane. The parameter $V$ is called visibility and is defined as:

$$
V=\frac{I_{\mathrm{Max}}-I_{\mathrm{Min}}}{I_{\mathrm{Max}}+I_{\mathrm{Min}}}
$$

with $I_{\mathrm{Max}}$ and $I_{\text {Min }}$ denoting the maximum and its consecutive minimum intensities of the interferogram. The visibility allows the inference of the synchrotron radiation complex degree of spatial coherence $\gamma$ using:

$$
\gamma=\frac{I_{1}+I_{2}}{2 \sqrt{I_{1} I_{2}}} V
$$

where $I_{1}$ and $I_{2}$ are the light intensities illuminating the pinholes 1 and 2, respectively, and which is related to the electron beam transverse profile. Applying the Van Citter-Zernike theorem, and assuming a Gaussian beam distribution along the pinhole axis, the projection of the beam size $\sigma$ over that axis is given by [5]:

$$
\sigma=\frac{\lambda L}{\pi D} \sqrt{\frac{1}{2} \log \left(\frac{1}{\gamma}\right)},
$$

where $L$ is the distance between the source point and the double-pinhole mask.

Note that if the radiation produced by each electron is considered as a spherical wave, the intensity of the light is uniformly distributed in all the directions, and $I_{1}=I_{2}$. In this case, the visibility $V$ coincides with $\gamma$. This condition is verified in the vertical direction if the angular divergence of the electron beam $\left(\sigma_{y}^{\prime}\right)$ is much lower with respect to the
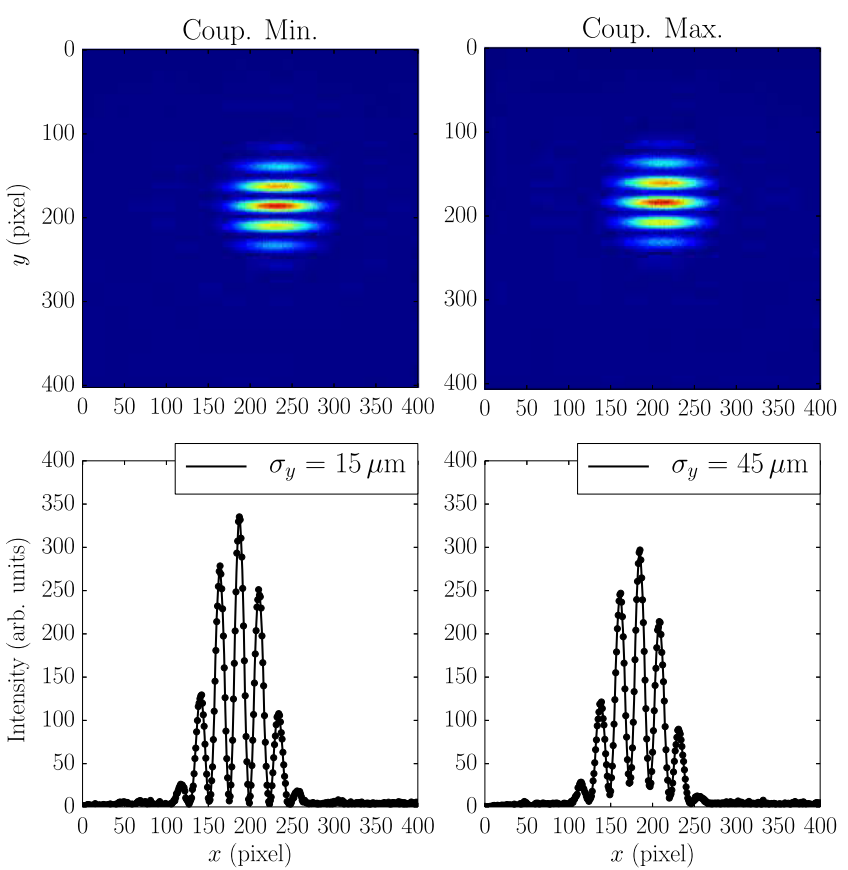

FIG. 4. Interferograms at minimum and maximum coupling used to measure the vertical beam size, and the data analysis to obtain the results. Black dots represent the projection of the central slice of the interferogram, while the black line is obtained fitting the data using Eq. (1). Note the different visibilities leading to different beam sizes.

angular divergence of the synchrotron radiation selected by the SRI system $(\eta)$. This condition is respected in the ALBA case, where $\sigma_{y}^{\prime}=0.37 \mu \mathrm{rad}$ (see Table I), and $\eta=$ $\frac{D}{L} \simeq 1 \mathrm{mrad}$ (see Table II).

In the ideal case of a single electron, located far from the SRI setup, and centered with respect to the double-pinhole system, the visibility of the interferogram would be strictly 1 . When considering an extended beam, the visibility is reduced due to a shift in the phase of the interferograms produced by the different electrons. As an example, Fig. 4 (top) shows two vertical interferograms at ALBA for minimum (left) and maximum (right) couplings. The different visibilities in the two images can be distinguished, especially by looking at the projection of the central slice (bottom plots in Fig. 4), where the minima for the maximum coupling case are larger than the minima for the minimum coupling case.

The beam sizes in Fig. 4 (15 and $45 \mu \mathrm{m}$, respectively) are obtained from Eq. (4), where $\gamma$ is calculated from the visibility inferred by fitting the projection of the central slice of the interferogram to Eq. (1), and the spherical wave approximation $\left(I_{1}=I_{2}\right)$ is assumed (and verified during measurements). Note that these values represent the projected beam size in the direction of the axis parallel to the double-pinhole system, but no information about possible beam tilts is given. Therefore, when the beam is tilted as in the ALBA case (recall Fig. 1), the horizontal and vertical 
beam size measurements performed using SRI are incomplete.

\section{BEAM RECONSTRUCTION}

Double-pinhole SRI provides the size of the projection of the beam shape along the pinholes axis. A method to fully reconstruct the beam using this technique has been developed. The assumption of the ellipticity can be exploited to reduce the number of samples needed to perform the measurement.

\section{A. Beam profile Rreconstruction}

A generic shape with a 2-axis symmetry, such as the beam ellipse, can be reconstructed measuring its projections $\sigma_{p}$ along different axis. This kind of measurement can be performed using double-pinhole SRI rotating the pinholes by a given angle $\theta$, which is equivalent to rotating the coordinate system by the same amount.

When tilting the double-pinhole axis by $\theta$, the interference fringes appear tilted as can be seen in Fig. 5. The projection $\sigma_{p}$ is inferred by analyzing the fringes in the direction of the rotating angle $\theta$. White lines in Fig. 5, represent the slice of the interferogram selected for the fitting process.

From each measurement at a given $\theta$, two parallel lines

$$
y=m x \pm Y_{0},
$$
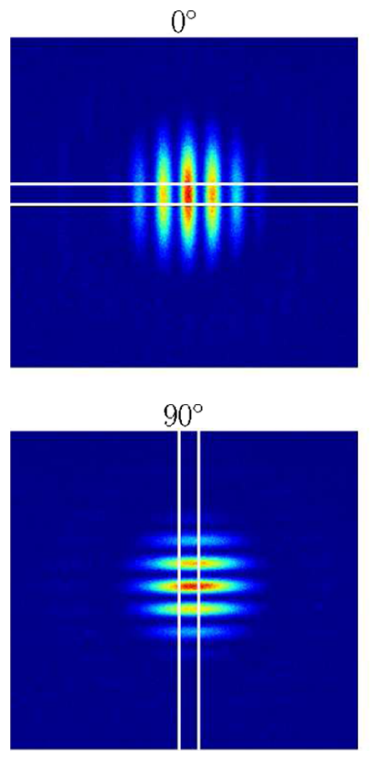

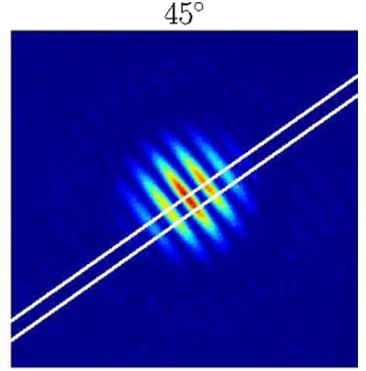

$135^{\circ}$

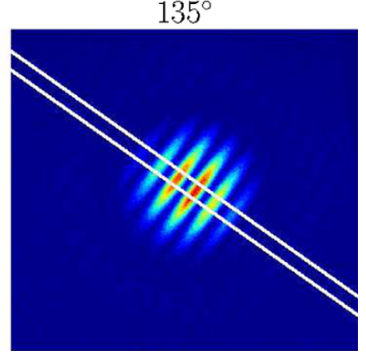

FIG. 5. Interferograms rotated at $0^{\circ}, 45^{\circ}, 90^{\circ}$, and $135^{\circ}$ to measure the size of the projection of the beam along the respective axis. White lines are oriented in the direction of the pinholes axis, rotated at angle $\theta$, and represent the slices selected for the fitting process.

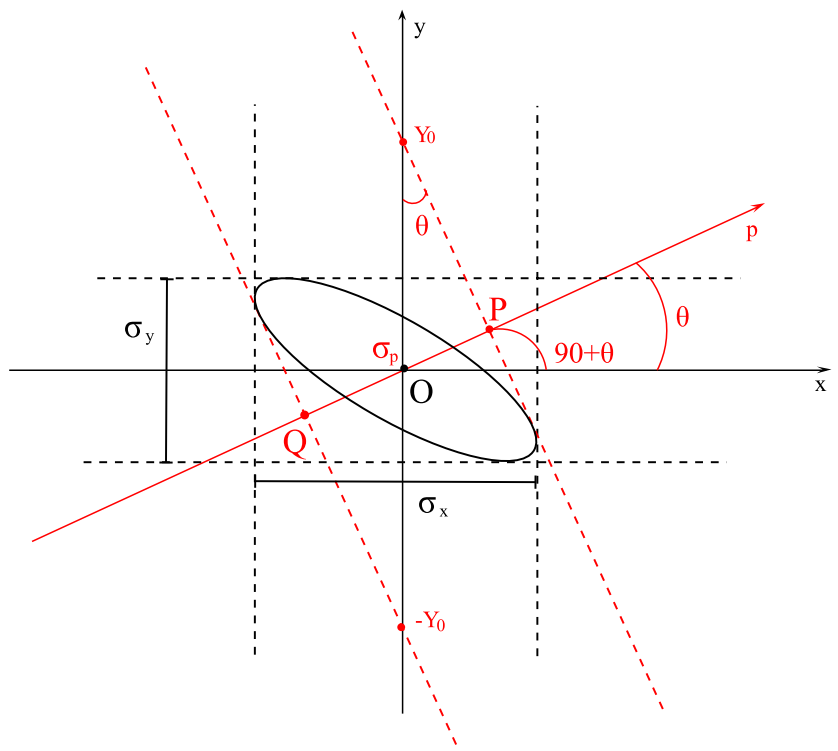

FIG. 6. Sketch of the transverse beam shape reconstruction using tangent lines calculated according to Eq. (6). Black lines represent the laboratory reference system, black dashed lines are drawn considering $\theta=0^{\circ}$ and $\theta=90^{\circ}$, and provide the projection along the $x$ and $y$ axis, $\sigma_{x}$ and $\sigma_{y}$. The red solid line represents the rotation direction of the double-pinhole axis of a generic angle $\theta$, while red dashed lines are the tangent lines calculated taking into account $\sigma_{p}$ (the distance PQ in the drawing).

tangents to the transverse beam shape, can be calculated as shown in Fig. 6. The slope of the lines, $m$, is given by the double-pinhole tilt angle $\theta$, while the position of the intersecting points between the tangent line and the $y$-axis, marked as $\pm Y_{0}$ are inferred from the triangle $O P Y_{0}$. The mathematical expressions are

$$
\left\{\begin{array}{l}
m=\tan \left(\theta+90^{\circ}\right) \\
\pm Y_{0}= \pm \frac{\sigma_{p}}{2 \sin \theta}
\end{array}\right.
$$

An example of beam reconstruction is presented in Fig. 7, top. Data have been taken rotating the pinhole system every $5^{\circ}$ in the range $\left[0^{\circ}, 200^{\circ}\right.$, the tangential lines are calculated using Eq. (6). This provides a "tomographic" reconstruction where the beam profile corresponds to the hollow shape enclosed by the black tangential lines. Obviously, the larger the number of tangent lines is, the more precise the beam shape can be reconstructed.

Next, a useful simplification assuming an elliptic beam is presented.

\section{B. Elliptic beam}

Assume that the beam can be described by the tilted ellipse shown in Fig. 8, with $\sigma_{u}$ and $\sigma_{v}$ the ellipse semi-axis, 

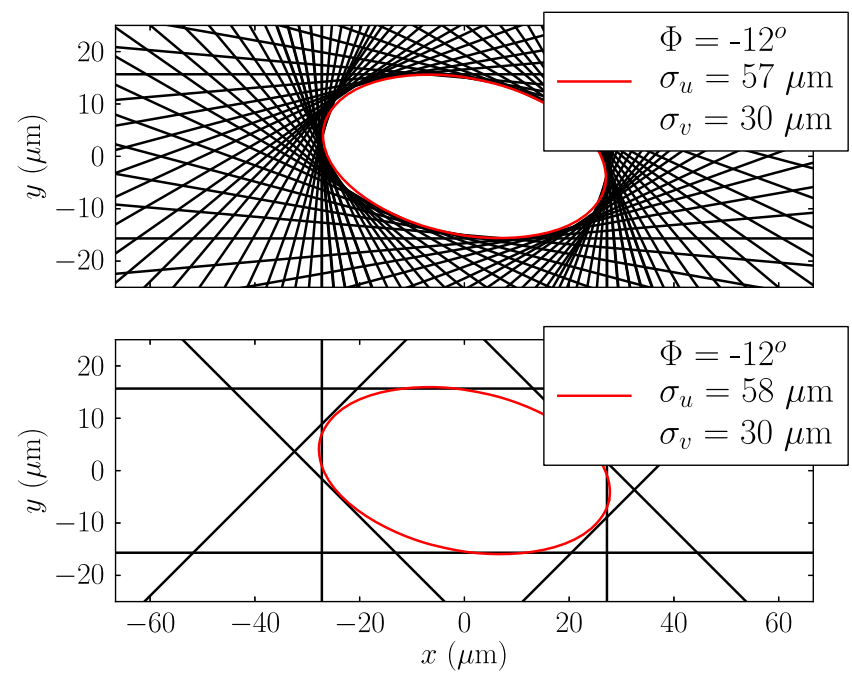

FIG. 7. In the first plot the full reconstruction of the transverse beam shape using tangents lines, in the second only the lines obtained by the projections at $0^{\circ}, 45^{\circ}, 90^{\circ}$, and $135^{\circ}$ are considered. The red ellipses are drawn using the results of the fit presented in Fig. 9.

and $\Phi$ the ellipse angle tilt. This ellipse is described by the general expression:

$$
a x^{2}+b y^{2}+2 c x y=1,
$$

where the parameters $(a, b, c)$ are

$$
a=\left(\frac{\cos ^{2} \Phi}{\sigma_{u}^{2}}+\frac{\sin ^{2} \Phi}{\sigma_{v}^{2}}\right)
$$

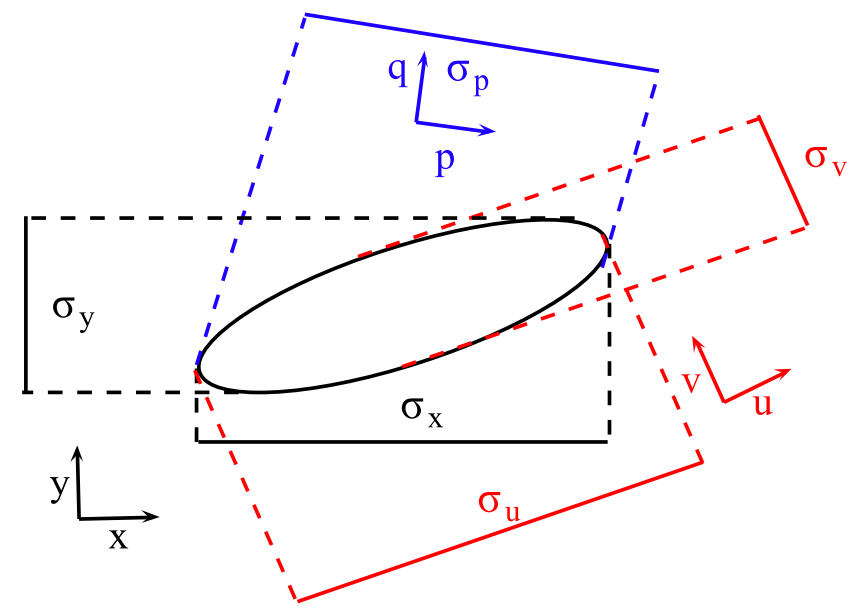

FIG. 8. General tilted ellipse: $(x, y)$ is the laboratory reference system (in black), $(u, v)$ refers to the reference system in which the ellipse is not tilted (in red), while $(p, q)$ refers to the one rotated by an angle $\theta$ with respect to the laboratory system (in blue).

$$
\begin{gathered}
b=\left(\frac{\sin ^{2} \Phi}{\sigma_{u}^{2}}+\frac{\cos ^{2} \Phi}{\sigma_{v}^{2}}\right) \\
c=\cos \Phi \sin \Phi\left(\frac{1}{\sigma_{v}^{2}}-\frac{1}{\sigma_{u}^{2}}\right) .
\end{gathered}
$$

For convenience, Eq. (7) has been written in analogy with the well-known phase space ellipse in the accelerator physics, where the parameter $b$ in this case corresponds to $\beta / \epsilon$ in Ref. [1], with $\beta$ the vertical beta function and $\epsilon$ the beam emittance. The horizontal projection can be expressed as:

$$
\sigma_{p}=\sqrt{\left(\frac{A}{\pi}\right) b}
$$

where $A$ corresponds to the ellipse area,

$$
A=\pi \sigma_{u} \sigma_{v} .
$$

Merging Eqs. (8c), (9), and (10), we find that the horizontal projection is

$$
\sigma_{x}=\sqrt{\sigma_{u}^{2} \cos ^{2} \Phi+\sigma_{v}^{2} \sin ^{2} \Phi} .
$$

Rotating the double-pinhole system by a known angle $\theta$ is equivalent to the rotation of the coordinate system by the same amount. In this new coordinate system, the tilt angle is now $\Phi-\theta$, and so the tilted projection is

$$
\sigma_{p}(\theta)=\sqrt{\sigma_{u}^{2} \cos ^{2}(\Phi-\theta)+\sigma_{v}^{2} \sin ^{2}(\Phi-\theta)}
$$

where $\sigma_{p}(\theta)$ is the projected beam size at the angle $\theta$.

The same data used to reconstruct the beam shape in Fig. 7 have been fitted using Eq. (12). The result is presented in the top plot of Fig. 9. This function fits to the data accurately, indicating a good agreement with the proposed model. At the maxima location the measurements look flatter with respect to the fit. This might be related with the real shape of the beam that is not exactly elliptic.

The red ellipse presented in Fig. 7 are drawn using the results of the fit presented in Fig. 9. From the same set of data, the four projections for $0^{\circ}, 45^{\circ}, 90^{\circ}$, and $135^{\circ}$ have been extracted. The data and fit using Eq. (12) are presented in the middle plot of Fig. 9. Also in this case the agreement is good: in the bottom plot of Fig. 9 the difference between the two fit curves as a percentage of the first $\left(\frac{\Delta \sigma}{\sigma}\right)$ is presented. The maximum deviation is lower than $1 \mu \mathrm{m}$, corresponding to the $0.7 \%$. This value is smaller with respect to the statistical error associated with standard SRI beam size measurements ( $\gtrsim 1 \mu \mathrm{m})$. 

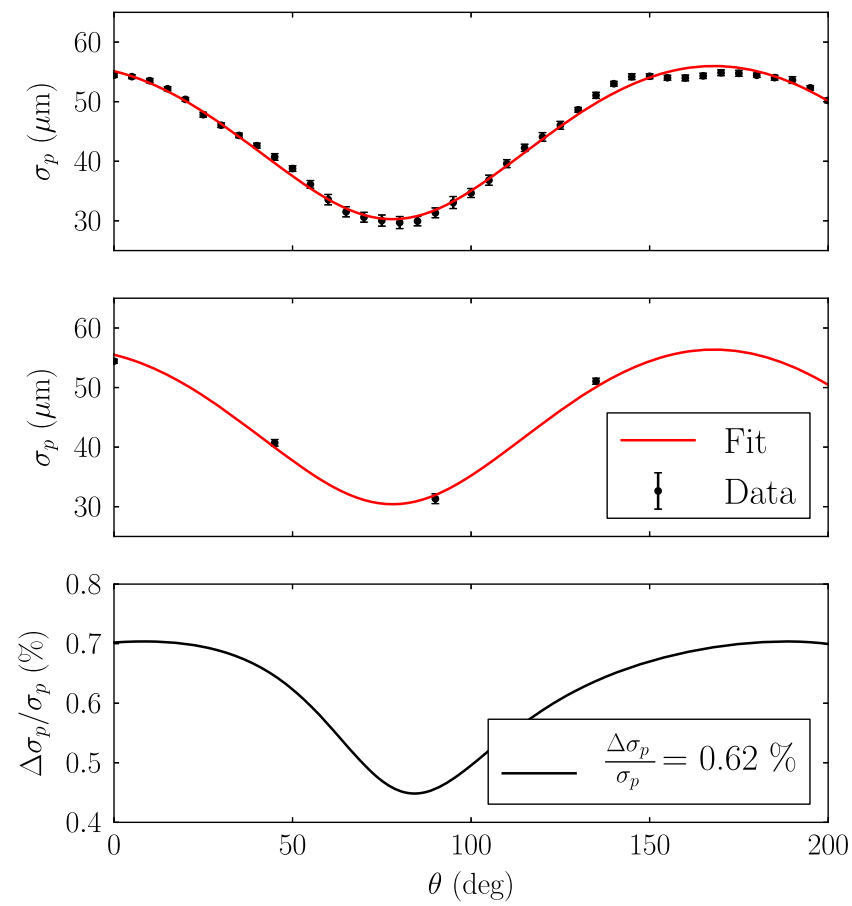

FIG. 9. Beam projection as a function of the pinhole rotation angle $\theta$. In the first plot all the 40 measurements, while in the second the projections at $0^{\circ}, 45^{\circ}, 90^{\circ}$, and $135^{\circ}$ are presented. The third plot (black line) is the difference between the fits obtained with 40 and 4 points as a percentage of the 40 points curve.

The same procedure has been applied to other quartets of projections taken at angles separated by $45^{\circ}$, starting from different initial angles $\theta_{0}$, i.e. $\theta_{0}=0^{\circ}$ refers to the quartet $\left[0^{\circ}, 45^{\circ}, 90^{\circ}, 135^{\circ}\right]$, while $\theta_{0}=5^{\circ}$ corresponds to $\left[5^{\circ}, 50^{\circ}\right.$, $\left.95^{\circ}, 140^{\circ}\right]$. The relative difference $\frac{\Delta \sigma}{\sigma}$ has been calculated for the different quartets and results are presented in

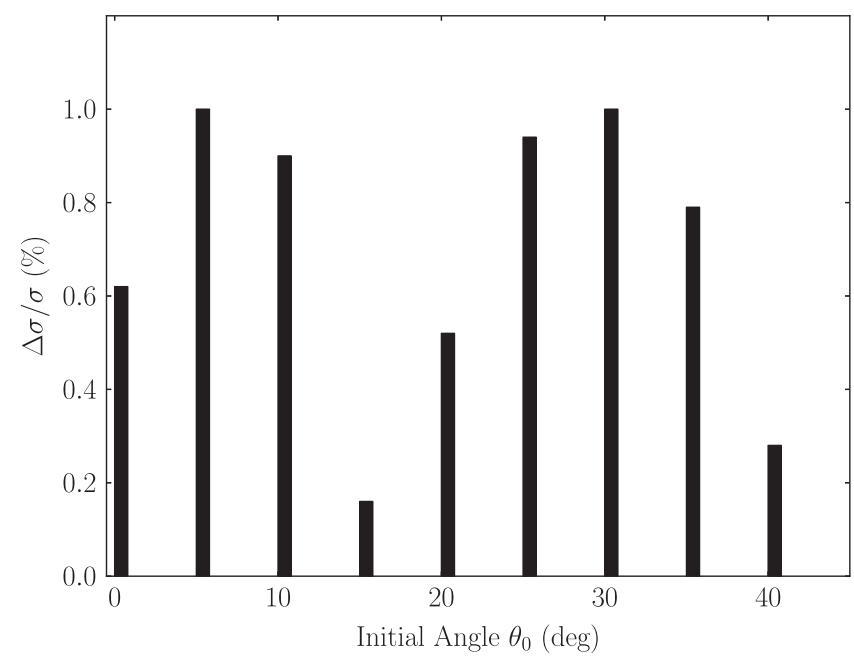

FIG. 10. Mean value of the difference between the curve obtained by fitting 200 projections and the one fitting only 4 projections as a percentage of the first.
Fig. 10. The mean deviation from the results obtained is always below the $1 \%$, which is comparable with the statistical error obtained during standard SRI measurements.

Note that this method allows us to properly reproduce the geometrical beam ellipse, since it provides its eigenvalues $\left(\sigma_{u}\right.$ and $\left.\sigma_{v}\right)$ and the beam tilt. Moreover, using the fitting method, only four projections are needed to reconstruct the full beam. This simplification allows us to speed up the measurements process.

\section{RESULTS FOR DIFFERENT COUPLINGS}

Measurements were performed at the ALBA diagnostic beamline Xanadu. The double-pinhole system is mounted on a graduated rotation stage in order to manually control the rotation angle $\theta$.

For each projection, 100 interferograms have been registered. The analysis of each interferogram has been performed by numerical rotation of the images by the pinholes axis inclination angle $(\theta)$, taking the projection of the central slice and fitting it using Eq. (1). The visibilities
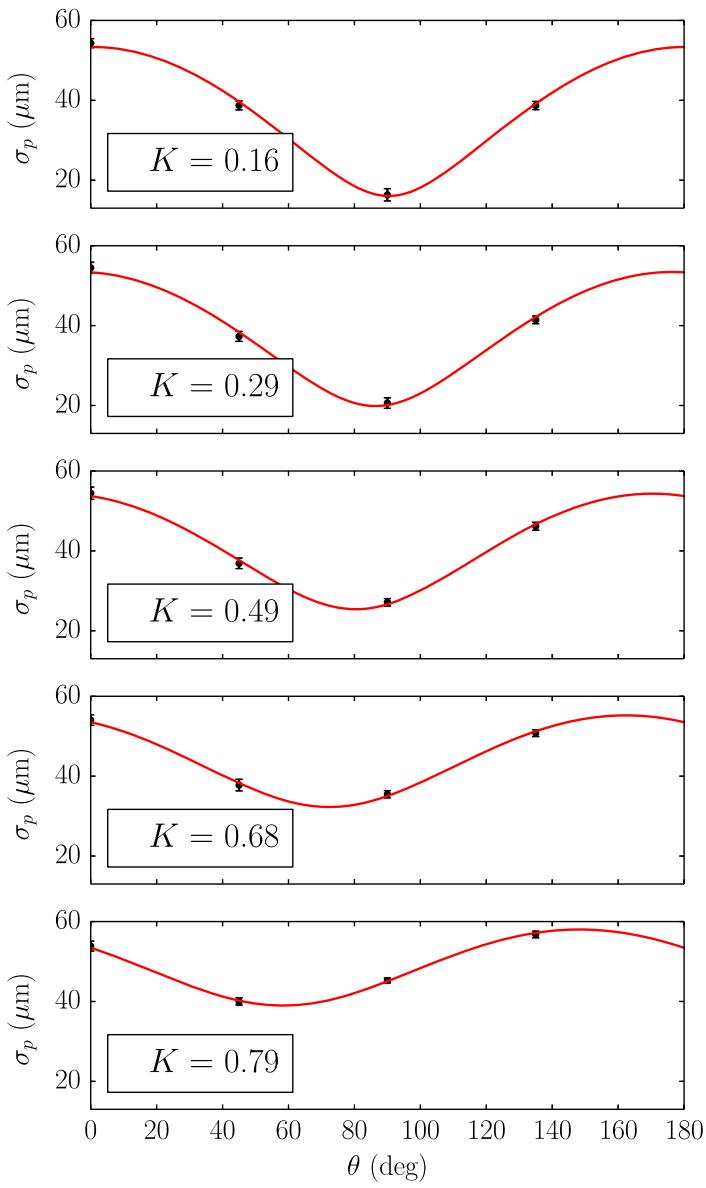

FIG. 11. Fit of projections taken at $0^{\circ}, 45^{\circ}, 90^{\circ}$, and $135^{\circ}$ to reconstruct the ellipse taken at different couplings $(K)$. Black dots are real data, while the red lines are obtained by fitting. 

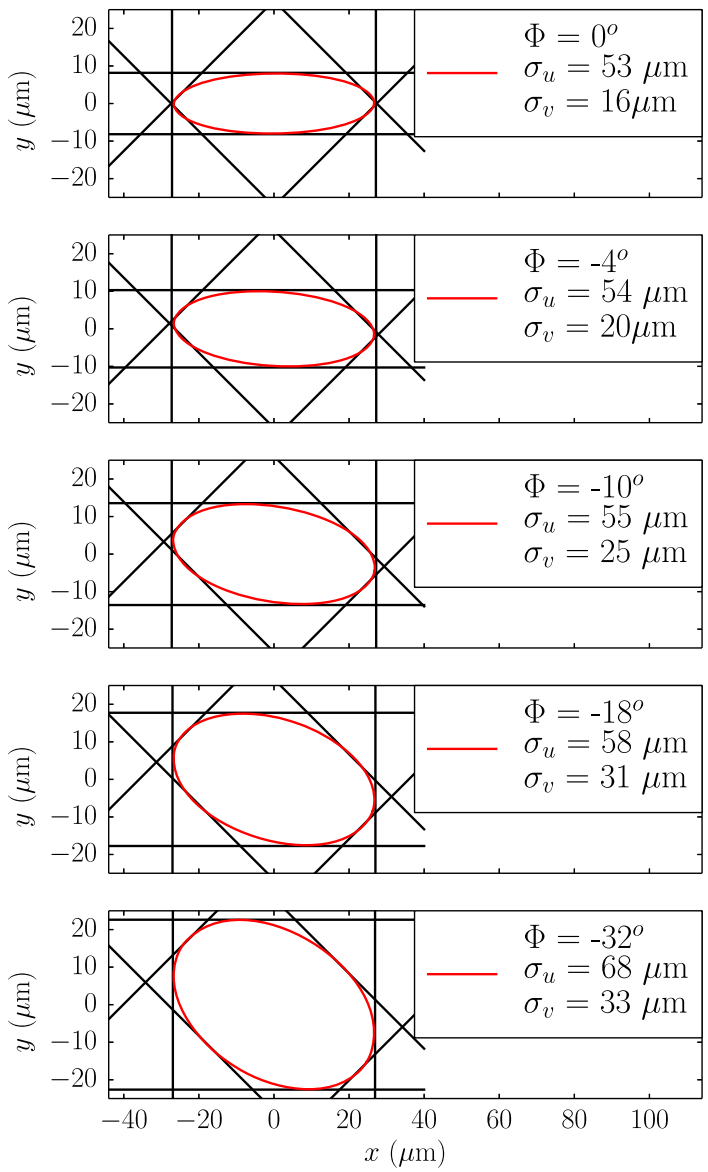

FIG. 12. Reconstruction of the beam at different couplings. Black lines are calculated from real data while the red ellipse is drawn from the result of the fit.

are used to compute the beam size using Eq. (4). The final result is the average value of the 100 measurements, and the error bar is purely statistical and is given by the standard deviation of the measurements.

In order to check the reconstruction technique consistency, measurements have been performed at five different values of the machine coupling, $K$, to change the tilt and the beam sizes.

The beam size values are measured rotating the double pinhole system at $0^{\circ}, 45^{\circ}, 90^{\circ}$, and $135^{\circ}$, using the double-pinhole SRI reconstruction. The fit results using Eq. (12) are presented in Fig. 11, while the reconstructed ellipses inside the tangent lines are presented in Fig. 12.

A machine optics analysis using the program "Linear Optics from Closed Orbits" (LOCO) [12,13] has been launched at each coupling in order to compare the results obtained by the measurements with the theoretical values. LOCO calculates a parametrized model response matrix and fits it to a measured matrix using a linear approximation method, from where the beam parameters at the Xanadu source point are inferred.
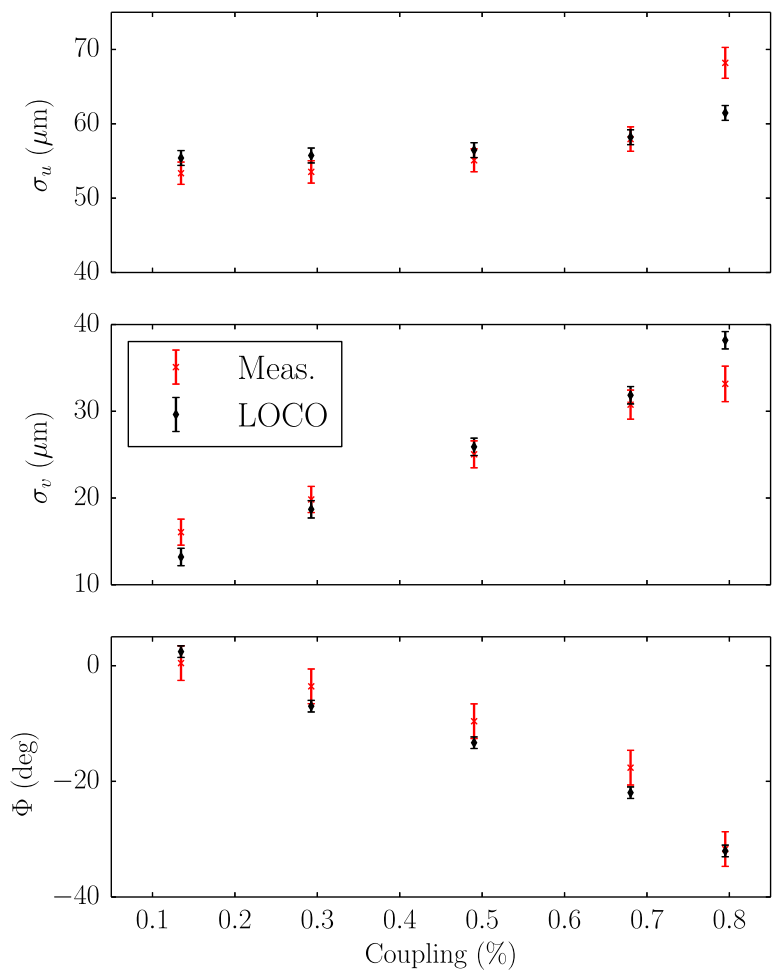

FIG. 13. Comparison of the SRI reconstruction with the results from LOCO for the horizontal and vertical beam size and the beam tilt at the Xanadu location.

Figure 13 presents the comparison of the SRI reconstruction with LOCO outputs. Results for the beam sizes are consistent at least for the first four values of the coupling, and only for the largest coupling $(0.8 \%)$ the LOCO value is outside the measurement error bar for both horizontal and vertical beam sizes. On the other hand, the measured tilt angle has some discrepancy, but always within the statistical error of the measurement. Taking into account the possible misalignments between the rotation stage and the $\mathrm{CCD}$, the experiments are considered satisfactory.

\section{ULTRA-SMALL BEAM SIZE MEASUREMENTS}

With the current skew quadrupole configuration at ALBA, the beam coupling can only be corrected to $0.15 \%$. However, other light sources could decrease this value to $0.01 \%$, corresponding to a beam size of $3.5 \mu \mathrm{m}$. At ALBA, measurements of such a small beam size would require a distance between pinholes of about $D \sim 60 \mathrm{~mm}$, which is actually impossible with the current half-mirror setup of the Xanadu beamline. Moreover, as mentioned in Ref. [11], this seems to be the smallest measurable size with the normal setup of the interferometer.

Also, in future light sources like ESRF-II or Sirius $[14,15]$, the vertical beam sizes will range between $\sim[2,10] \mu \mathrm{m}$ depending on the coupling correction. 


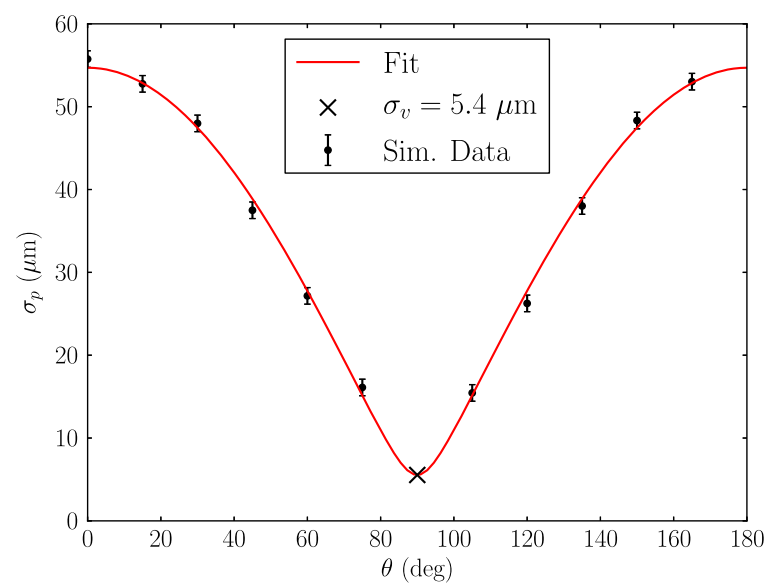

FIG. 14. Simulated $5 \mu \mathrm{m}$ vertical beam size reconstruction using the rotated SRI technique using SRW. Black dots are the SRW simulated data, the error bar is fixed to $1.5 \mu \mathrm{m}$, and red line is the result of the fit using Eq. (12). The black cross indicates the result from the fit for the $\sigma_{v}$.

To overcome possible limitations for these cases, one can perform double-pinhole SRI using the "rotating mask" method and, rather than actually measuring the smallest (vertical) beam size with the standard SRI, infer this parameter from Eq. (12), using the beam profile reconstruction described previously.

\section{A. SRW simulations results}

Simulations have been performed using Synchrotron Radiation Workshop (SRW) [16] to study the feasibility of the rotating mask technique applied to ultrasmall beam size measurements. Simulations are performed using the ALBA machine parameters (Table I), and propagating the generated synchrotron radiation through the ALBA diagnostic beamline Xanadu. An elliptic beam with a horizontal beam size (major ellipse semiaxis) of $55 \mu \mathrm{m}$ has been generated. The vertical beam size (minor ellipse semiaxis) has been changed from 2 to $10 \mu \mathrm{m}$, and the tilt angle left at 0 for simplicity. To perform the beam reconstruction, projection measurements have been simulated sampling the angle in steps of $15^{\circ}$, skipping $\theta=90^{\circ}$, which corresponds to the minimum beam size.

Finally, interferograms have been analyzed and the projected beam sizes as a function of the angles have been fitted using Eq. (12). The results of the fits provides the beam ellipse parameters. As an example, Fig. 14 presents the results obtained by simulating a nontilted beam with horizontal and vertical beam sizes of $55 \mu \mathrm{m}$ and $5 \mu \mathrm{m}$ respectively. In this case, a value of $\sigma_{v}=5.4 \mu \mathrm{m}$ is obtained from the fit, compatible with the initial input parameter.

Results for the different vertical beam sizes are shown in Table III. Note that some of the beam sizes are smaller than the theoretical expected value. This is not possible when performing standard SRI, since the visibility can only be reduced (and consequently the beam size increased) by all the possible sources of noise. This is because the parameter is not inferred by direct SRI measurements, but from the secondary fit using Eq. (12). A study of the error of the measurements is presented next.

\section{B. Error study}

In order to understand the limit of the SRI reconstruction method for ultrasmall beam sizes, a systematic study on the error that might affect the fit has been performed, where the SRW simulations presented in the previous subsection have been taken as a starting point. Each reconstruction is composed of beam projections sampled for 11 rotation angles, $\theta$.

When performing real measurements at the Xanadu diagnostic beamline, each SRI projected beam size measurement fluctuates around $1.5 \mu \mathrm{m}$. To simulate these fluctuations, each $\sigma_{p}$ used for the reconstruction has been smeared by a random value generated according a normal distribution centered in 0 and with standard deviation given by the expected statistical error (in this case $0.5,1,1.5$, and $2 \mu \mathrm{m}$. See Table III). For each beam size the smearing and reconstruction process has been repeated 100 times. The average of the results of this process and the standard deviation provide the final result and associated error for the beam size.

The results for the different beam sizes are presented in Table III and Fig. 15: the smaller the smearing statistical error, the smaller the error associated with the final reconstruction. For beam sizes of $2 \mu \mathrm{m}$, even the smallest deviation $(0.5 \mu \mathrm{m})$ leads to fluctuations in the beam size reconstruction of $85 \%$ of expected value. However, note that the average of several measurements provides for all

TABLE III. Results of simulations of small vertical beam size reconstruction: rotating SRI reconstruction without smearing compared with results produced by smearing the rotated beam sizes of $0.5,1,1.5$, and $2 \mu \mathrm{m}$ with the respective error.

\begin{tabular}{lccccc}
\hline \hline & \multirow{2}{*}{$\sigma_{y}$ reconstructed $(\mu \mathrm{m})$} & \multicolumn{3}{c}{ Smeared reconstructed $\sigma_{y}(\mu \mathrm{m})$} \\
\cline { 2 - 5 }$\sigma_{y}$ Theoretical $(\mu \mathrm{m})$ & Not Smeared & Simulated $0.5 \mu \mathrm{m}$ & Simulated 1 $\mu \mathrm{m}$ & Simulated 1.5 $\mu \mathrm{m}$ & Simulated 2 $\mu \mathrm{m}$ \\
\hline 2 & 2.2 & $2.3 \pm 1.7$ & $2.6 \pm 2.3$ & $2.5 \pm 2.8$ & $3.3 \pm 3.4$ \\
5 & 5.4 & $5.3 \pm 1.1$ & $5.7 \pm 1.6$ & $5.7 \pm 2.1$ & $6.5 \pm 2.9$ \\
7 & 6.9 & $7.0 \pm 0.8$ & $6.6 \pm 1.7$ & $6.9 \pm 1.9$ & $7.5 \pm 2.4$ \\
10 & 9.7 & $9.7 \pm 0.6$ & $9.5 \pm 1.4$ & $9.6 \pm 2.1$ & $9.3 \pm 2.5$ \\
\hline \hline
\end{tabular}



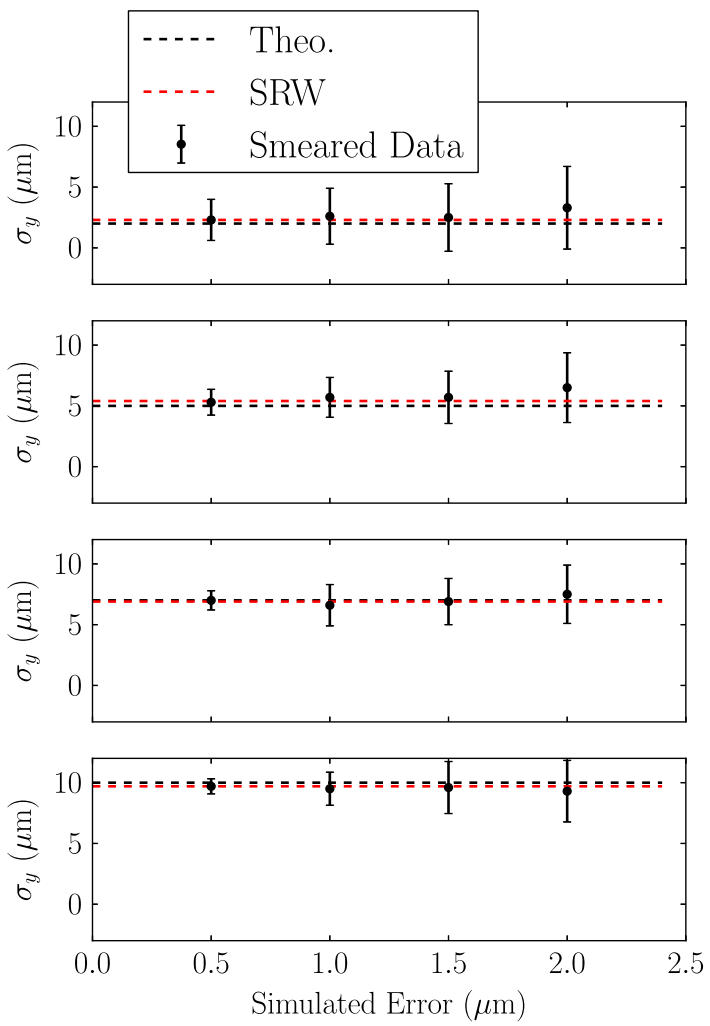

FIG. 15. Results for $2 \mu \mathrm{m}, 5 \mu \mathrm{m}, 7 \mu \mathrm{m}$, and $10 \mu \mathrm{m}$ beam size reconstruction for SRI beam size measurement statistical errors of $0.5 \mu \mathrm{m}, 1 \mu \mathrm{m}, 1.5 \mu \mathrm{m}$, and $2 \mu \mathrm{m}$. Black dots are the obtained values, the error bar is given by the standard deviation of 100 smeared reconstructions, the black dashed line is the theoretical value, while the red dashed line is the result obtained performing rotating SRI SRW simulations.

the simulated beam sizes a result compatible with the expectations, improving the standard SRI resolution.

The standard fluctuation obtained when measuring the ALBA beam size is around $1.5 \mu \mathrm{m}$, mainly due to the Xanadu optical components vibrations. However, other SRI measurements, performed at more robust beamlines, using for example granitic supports for optical elements, or more direct in-vacuum light paths to avoid airvibrations, such as the one described in Ref. [17], might produce sub-micrometer errors. This test underlines the importance of designing the SRI measurements in robust beamlines, in order to obtain a better accuracy on ultrasmall beam size measurements.

\section{CONCLUSIONS}

This paper shows a method of performing full transverse beam profile reconstruction using visible light SRI. It is based on the rotation of the mask holding the double-pinhole system, and measures the projected beam size at different rotating angles using the standard SRI.
Assuming the beam has an elliptic shape, we show that four projections already provide a reliable value for the beam eigenvalues. This method has been tested at ALBA under different coupling conditions, and the results are consistent with the expectations within $1.5 \mu \mathrm{m}$ over a minimum vertical beam sizes of $16 \mu \mathrm{m}$. Furthermore, we show how this method can be used to infer ultrasmall beam sizes (up to $2 \mu \mathrm{m}$ ), without actually measuring the smallest beam projection (typically corresponding to the vertical plane). SRW simulations have been performed to validate this technique and calculate associated uncertainties, showing how this technique can become a useful tool for future beam size measurements.

\section{ACKNOWLEDGMENTS}

The authors want to acknowledge the ALBA accelerator division, and in particular Z. Martí for the help with LOCO. This work owes a lot to T. Mitsuhashi, for the valuable help in developing the SRI in its first stage. Finally a special thanks to all the ALBA staff for the efforts to maintain and improve the facility.

[1] H. Wiedemann, Particle Accelerator Physics (Springer, Berlin, 2007).

[2] G. Kube, Review of synchrotron radiation based diagnostics for transverse profile measurements, in Proceedings of 8th European workshop on beam Diagnostic and Instrumentation of Particle Accelerator (DIPAC 2007), Venice, Italy, 2007 (JACoW, Geneva, 2007), p. 6, number MOO1A03.

[3] P. Elleaume, C. Fortgang, C. Penel, and E. Tarazona, Measuring beam sizes and ultrasmall electron emittances using an X-ray pinhole camera, J. Synchrotron Radiat. 2, 209 (1995).

[4] U. Iriso, M. Alvarez, F. Fernandez, A. Olmos, and F. Pérez, Diagnostics during the ALBA storage ring commissioning, in Proceedings of 11th European workshop on beam Diagnostic and Instrumentation of Particle Accelerator (DIPAC 2010), Hamburg, Germany, 2010 (JACoW, Geneva, 2010), p. 280, number TUOA02.

[5] T. Mitsuhashi, Beam profile and size measurement by SR interferometers, in Beam Measurements, Proceedings of Joint US-CERN-Japan-Russia School on Particle Accelerators, Montreux and Geneva, Switzerland, 1998 (World Scientific, Singapore, 1998).

[6] L. Torino and U. Iriso, Beam size measurements using synchrotron radiation interferometry at ALBA, in Proceedings of $3 \mathrm{rd}$ International Beam Instrumentation Conference (IBIC 2014), Monterrey, USA, 2014 (JACoW, Geneva, 2014), p. 428, number TUPF23.

[7] L. Torino and U. Iriso, Limitations and solutions of beam size measurements via interferometry at ALBA, in Proceedings of 4th International Beam Instrumentation Conference (IBIC 2015), Melbourne, Australia, 2015 (JACoW, Geneva, 2015), p. 428, number TUPB049. 
[8] M. Masaki and S. Takano, Two-dimensional visible synchrotron light interferometry for transverse beam-profile measurement at the SPring-8 storage ring, J. Synchrotron Radiat. 10, 295 (2003).

[9] C. L. Li, J. Corbett, Y. H. Xu, M. J. Boland, T. Mitsuhashi, W. J. Zhang, and M. Grinberg, Double-slit interferometer measurements at Spear-3, in Proceedings of 7th International Particle Accelerator Conference (IPAC 2016), Busan, South Korea, 2016 (JACoW, Geneva, 2016), p. 368, number MOPMR054.

[10] U. Iriso and F. Fernández, ALBA Project Document Report No. AAD-FE-DI-VMIR-01, 2011.

[11] T. Mitsuhashi, Recent trends in beam size measurements using the spatial coherence of visible synchrotron radiation, in Proceedings of 6th International Particle Accelerator Conference (IPAC 2015), Richmond, USA, 2015 (JACoW, Geneva, 2015), p. 3662, number THYC2.

[12] J. Safranek, Experimental determination of storage ring optics using orbit response measurements, Nucl. Instrum. Methods Phys. Res., Sect A 388, 27 (1997).
[13] G. Benedetti, D. Einfeld, Z. Martí, and M. Munoz, LOCO in the ALBA storage ring, in Proceedings of the 2nd International Particle Accelerator Conference, San Sebastiáán, Spain (EPS-AG, Spain, 2011), p. 2055, number WEPC024.

[14] F. Ewald, Challenges for emittance diagnostics for the ESRF low emittance lattice, in Presentation of 2 nd Diagnostic Expert of European Light Sources (DEELS 2015), Barcelona (Spain), 2015 (JaCoW, Geneva, 2015).

[15] In Sirius Light Source, http://lnls.cnpem.br/sirius-newbrazilian-synchrotron-light-source/.

[16] O. Chubar and P. Elleaume, Accurate and efficient computation of synchrotron radiation in the near field region, in Proceedings of the 6th European Particle Accelerator Conference, Stockholm, 1998 (IOP, London, 1998), p. 1177, number C980622.

[17] J. Breunlin, A. Andersson, Á. Saá Hernández, M. Rohrer, and N. Milas, Status of the new beam size monitor at SLS, in Proceedings of 5th International Particle Accelerator Conference (IPAC 2014), Dresden, Germany, 2014 (JACoW, Geneva, 2014), p. 3662, number THPME169. 